\title{
IMPLEMENTAÇÃO, TESTES E AVALIAÇÃO DO MÉTODO SASW (Spectral Analysis of Surface Waves)
}

Adriano Marchioreto ${ }^{1}$ (altaresolucao@altaresolucao.com.br) \& Fábio Taioli²

${ }^{1}$ Alta Resolução Geologia e Geofísica

${ }^{2}$ Instituto de Geociências da USP

Copyright 2004, SBGf - Sociedade Brasileira de Geofísica

Este texto foi preparado para a apresentação no I Simpósio Regional da Sociedade Brasileira de Geofísica, São Paulo, 26-28 de setembro de 2004. Seu conteúdo fol revisado pela Comissão Tecno-científica do I SR-SBGf mas não necessariamente representa a opinião da SBGf ou de seus associados. E proibida a reprodução total ou parcial deste material para propósitos comerciais sem prévia autorização da SBGt.

\section{Resumo}

O método "Spectral Analysis of Surface Waves" (SASW) é um método não destrutivo, baseado na geração e detecção de ondas elásticas, e o estudo da natureza dispersiva desta onda.

Como a natureza dispersiva da onda se dá em termos da freqüência, o processamento dos sinais é realizado no domínio da freqüência vem daí o no nome SASW.

O método SASW envolve medidas de ondas de superfície do tipo Rayleigh e é realizado na superfície, não necessitando de furos, tornando-se assim mais baratos do que os dois métodos sísmicos mais usados para o estudo de parâmetros elásticos do solo que são o "crosshole" e o "downhole". Ambos os métodos envolvem a análise de ondas de corpo $\mathrm{P}$ e $\mathrm{S}$, e requer a instalação de furos, consumindo tempo e custo.

A partir das velocidades de propagação das ondas elásticas $\mathrm{P}$ e $\mathrm{S}$ é possível determinar os parâmetros elásticos dos materiais (módulo de Young, coeficiente de Poisson, módulo de rigidez, etc.). Embora o SASW amostre um volume maior do terreno, a comparação com os resultados obtidos pelo método tradicional como o crosshole se faz necessária para medir a eficiência do método SASW na determinação das velocidades das ondas S no meio.

\section{Introdução}

Apesar de estudos revelarem que cerca de dois terços de toda energia de uma onda sísmica se propagar na forma de ondas de superfície, estas ondas são consideradas indesejáveis (ruídos) na maioria dos métodos sísmicos.

O método "Spectral Analysis of Surface Waves" (SASW) é uma técnica relativamente nova em que, através da determinação da dispersão das ondas de superfície Rayleigh, pode-se determinar perfis de velocidade das ondas $S$ e, a partir delas, os parâmetros elásticos do meio. Embora o método tenha mostrado bons resultados em algumas aplicações no exterior, até agora não se tem notícia de sua utilização no Brasil.

O ponto chave do método é a geração e detecção de ondas de superfície e o estudo da natureza dispersiva desta onda. Em um meio estratificado, a velocidade de propagação da onda de superfície depende de sua freqüência. $A$ variação da velocidade com a freqüência é chamada de dispersão e ocorre ondas com diferentes freqüências (ou comprimentos de ondas) amostrarem diferentes partes do meio. Ondas com altas freqüências (pequenos comprimentos de onda) se propagam somente nas camadas mais rasas, enquanto ondas com menores freqüências (maiores comprimentos de onda) se propagam desde camadas mais rasas até as mais profundas.

\section{Metodologia/ Problema Investigado}

O método SASW pode ser dividido entre três etapas:

A) Aquisição dos dados de campo (figura 1)

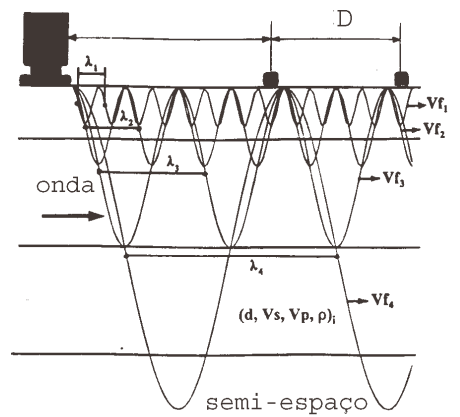

Figura 1 - Geração e aquisição das ondas de superfície.

B) Determinação da curva de dispersão das ondas Rayleigh

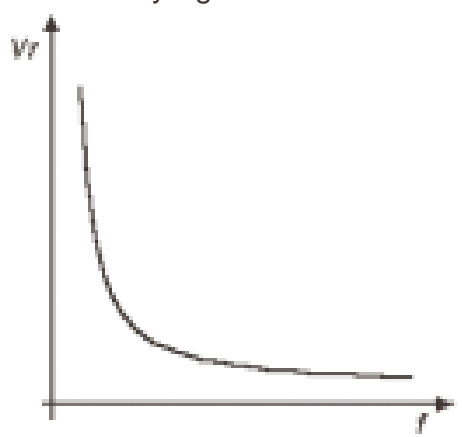

Figura 2 - Análise do Sinal - Curva de dispersão, velocidade de fase das ondas Rayleigh.

C) Inversão da curva de dispersão para obter um perfil de velocidade da onda $S$ 


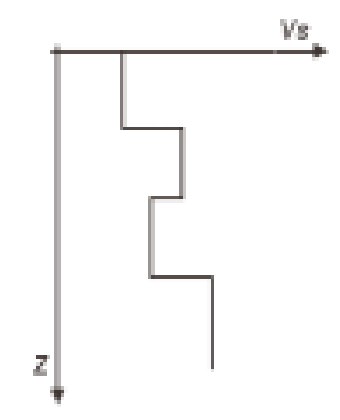

Figura 3 - Processo de inversão. Obtenção da velocidade da onda S.

Para cada uma das etapas foi feita uma descrição dos procedimentos, dando especial ênfase ao tópico b, que é o calculo das curvas de dispersão. É nesta etapa que se encontram os maiores problemas do método.

\section{Resultados}

A fim de avaliar a metodologia selecionaram-se três estudos de casos, que ilustram os principais aspectos da aplicação do método. Uma vez que o objetivo do trabalho era implementar, testar e avaliar a metodologia, estes três casos selecionados abrangem vários dos aspectos da aplicação prática do método.

O primeiro caso as curvas de dispersão obtidas para diferentes espaçamentos de geofones se sobrepõem numa determinada faixa de freqüência. $O$ segundo exemplo é um caso intermediário, onde as curvas variam pouco de uma para outra. No terceiro exemplo, as curvas para diferentes espaçamentos entre geofones não convergem, foi necessário tentar uma nova estratégia para confeccionar a curva de dispersão representativa da área.

Por fim a terceira etapa consiste na inversão da curva de dispersão. Diversos modelos iniciais foram gerados e todos eles após algumas dezenas de iterações acabam apresentando uma mesma tendência. No caso do campus da UNICAMP os valores concordam bem até 4 metros de profundidade com os valores de velocidade obtidos pelo crosshole, para profundidades maiores do que 4 metros os valores divergem. No caso da UNESP (Bauru) os valores de ambos os métodos divergem totalmente.

No campus da USP (São Paulo) há uma sobreposição praticamente perfeita nas curvas de dispersão geradas pelos diferentes espaçamentos entre geofones usados (Figura 4).

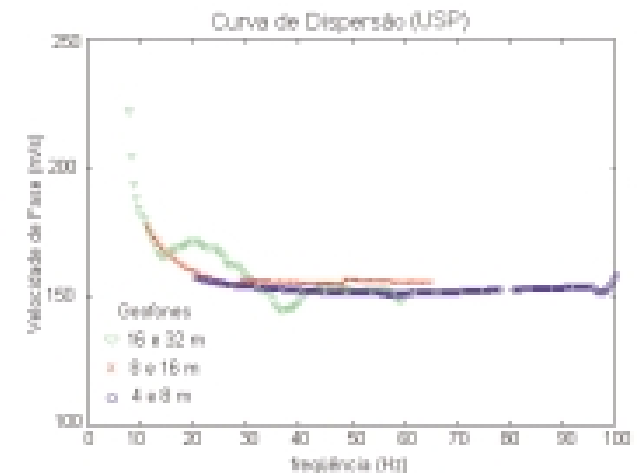

Figura 4 - Curvas de dispersão geradas por diferentes espaçamentos de geofones (Campus da USP).

Uma coincidência com esse grau de sobreposição não é comum; em geral, as curvas se comportam semelhantemente às obtidas no campus da UNICAMP (Figura 5).

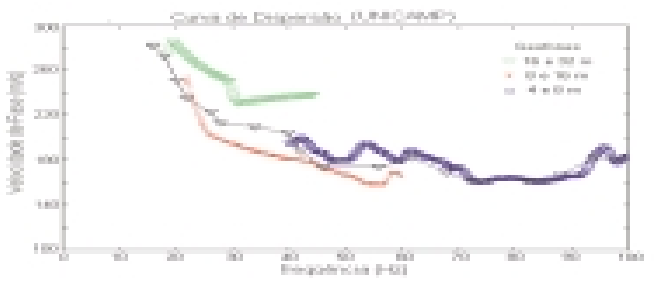

Figura 5 - Curvas de dispersão geradas por diferentes espaçamentos de geofones (Campus da UNICAMP).

Através da curva de dispersão mostrada na Figura 5 foi realizado um processo de inversão, de modo a gerar perfis de velocidade da onda S.

Os perfis obtidos a partir de diversos modelos iniciais acabaram convergindo para uma mesma tendência. Na Figura 6 são apresentados alguns perfis obtidos durante a inversão; na mesma figura estão os valores de um ensaio "crosshole" realizado na mesma área, por Carvalho et al., (2000).

Até aproximadamente $4 \mathrm{~m}$ de profundidade os resultados obtidos por ambos os métodos são muito semelhantes, com diferença sempre menor do que 10 $\%$. Abaixo de $4 \mathrm{~m}$ os valores encontrados são totalmente discordantes, e a exemplo do que ocorreu no trabalho de Wills (1998), os valores de velocidade obtidos pelo SASW são bem maiores do que as obtidas pelo "crosshole". 


\section{IMPLEMENTAÇÃO, TESTES E AVALIAÇÃO DO MÉTODO SASW} (Spectral Analysis of Surface Waves)

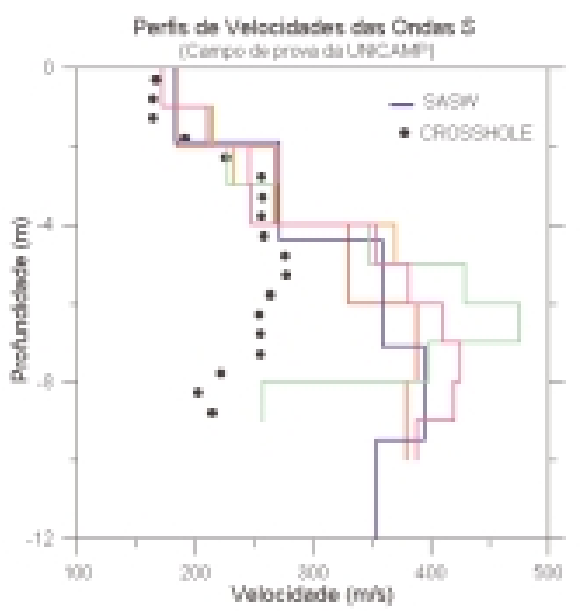

Figura 6 - Perfis de velocidades das ondas $S$ obtidos pelos ensaios SASW no campo de provas da UNICAMP, Campinas, SP.

No campus da UNESP (Bauru) foi o caso extremo; vários ensaios foram realizados e muitos deles apresentaram baixa relação sinal-ruído num amplo intervalo de freqüências; outros ensaios que possuíam aceitáveis coerências entre os sinais, foram impossíveis de interpretar, uma vez que o espectro de fase era totalmente aleatório, e em outros casos não existia correlação nos valores das curvas de dispersão entre os vários geofones (Figura 7).

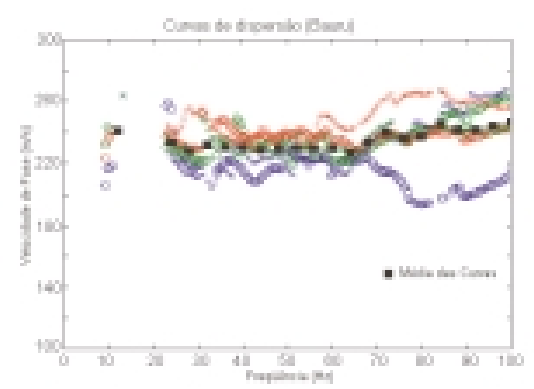

Figura 7- Curvas de dispersão geradas por diferentes espaçamentos de geofones (Campus da UNESP Bauru).

A dificuldade em gerar sinais com elevada energia em baixas freqüências é a maior causadora de problemas do método, uma vez que a baixa razão sinal-ruído torna o método sempre suscetível ao julgamento de um intérprete.

Outro problema encontrado na geração de energia por uma fonte impulsiva, é que seu comportamento não coerente muitas vezes ocasiona vales em determinadas freqüências dos sinais, sendo justamente nestas freqüências que 0 algoritmo que executa 0 desdobramento da fase falha, gerando saltos que, posteriormente, refletirão no cálculo da velocidade de fase.

O processo de desdobramento ("unwrap") da fase é um passo fundamental na determinação da curva de dispersão. Os algoritmos usados para calcular a FFT representam a fase em radiados, variando de $-\pi$ a $+\pi$. Para se ter uma visualização da fase completa é necessário haver um desdobramento da fase ("unwrap"). Este passo é extremamente importante pois, a partir da fase, pode-se calcular o tempo de atraso em função da freqüência. O principal problema está na geração de saltos fictícios na fase devido a presença ruídos no sinal. Para corrigir tais irregularidades é necessário julgamento e intervenção do intérprete, tornando o procedimento subjetivo.

Em sua tese Foti (2000) usou fonte de energia coerente (vibrador) e em determinados locais não conseguiu obter uma boa razão sinal-ruído para geofones posicionados a 10 e $20 \mathrm{~m}$ da fonte. O fato de não se obter elevada energia nas baixas freqüências é o maior limitante da profundidade investigada pelo método.

Outro aspecto a ser abordado nesta discussão é a inerente necessidade de processar todo o sinal registrado. Para as distâncias envolvidas no método, é praticamente impossível separar as ondas de superfície das ondas de corpo, como é realizada no processamento de sismos, uma vez que tanto no domínio do tempo, quanto no domínio da freqüência existe uma sobreposição dessas ondas. Os efeitos causados pelas ondas de corpo no estudo da superfície são tratados em alguns artigos como (Sanches-Salinero et al., 1987 e Tokimatsu, 1995). Estes efeitos são chamados de efeitos de proximidade ("near-field") e efeitos de interferência das ondas de corpo ("body wave interference effects"). Embora vários autores reconheçam tais problemas, as recomendações para atenua-los variam consideravelmente (Zywicki, 1999).

Estes diferentes critérios usados por vários autores evidenciam que não existe um consenso em torno dos efeitos causados pelas ondas de corpo. $\mathrm{Na}$ verdade, estes critérios são conflitantes, como pode ser observado nos critérios sugeridos por Heisey et al. (1982a) e por Sanches-Salinero et al. (1987).

Mesmo com esses problemas o uso de ondas de superfície Rayleigh com 0 intuito de investigações geotécnicas possui grande potencial e tem muito a ser desenvolvido, principalmente pelo fato que dois terços do total de energia liberada por uma fonte é transmitida através das ondas Rayleigh

Atualmente vem ganhando grande impulso o MASW ("Multichannel Analysis of Surface Waves") desenvolvido por (Park et al., 1999a e 1999b), Foti (2000) e Xia et al., (2000).

Nesta nova metodologia, as curvas de dispersão são obtidas através de filtro $f-k$, não existindo a passagem mais crítica do SASW, que é a necessidade do desdobramento da fase; conseqüentemente a obtenção da curva de dispersão pode ser facilmente automatizada, eliminando assim a participação do intérprete e reduzindo o tempo de interpretação e obtendo curvas de dispersão mais estáveis.

\section{Discussão e Conclusões}

Vários testes foram realizados no transcorrer desta pesquisa, chegando-se à conclusão que o método sofre de diversas limitações. Uma delas se deve à 
incapacidade de gerar boas curvas de dispersão em ambientes onde a relação sinal-ruído é baixa.

O uso de fonte impulsiva como marreta ou queda de peso mostrou não gerar energia em freqüências suficientemente baixas, fato este que ocasiona dois efeitos imediatos: a) diminuição da profundidade investigada e b) problemas na obtenção da fase do espectro cruzado. A baixa relação sinal-ruído para as menores freqüências é o principal fator impeditivo para tornar o método automático. Em adição, torna o método subjetivo, uma vez que necessita do julgamento de um intérprete.

No estudo de estruturas rasas, os valores de velocidade de ondas S obtidos concordam numa ordem de $10 \%$ com os dados de "crosshole"; à medida que se aumenta a profundidade, os valores obtidos pelos dois métodos divergem. Além da baixa energia nas baixas freqüências, outro fator que pode explicar essa diferença nas velocidades obtidas pelos dois métodos é devido aos efeitos causados pelas interferências das ondas de corpo, principalmente as ondas de corpo refratadas e refletidas.

Os ensaios realizados durante a pesquisa foram condensados em três casos que mostram tanto os aspectos positivos, quanto os negativos do método. Em dois dos casos mostrados pode-se fazer uma correlação direta com os dados de crosshole. Para o levantamento efetuado no campo de provas da UNICAMP os resultados divergem de menos de $10 \%$ até $4 \mathrm{~m}$ de profundidade, sendo que os resultados de ensaio "crosshole" indicam um aumento abrupto na velocidade em torno da profundidade de $2 \mathrm{~m}$, tendência detectada também pelo SASW, mostrando que realmente há um aumento de velocidade para esta profundidade. Para profundidades maiores do que $4 \mathrm{~m}$ os valores de velocidades dos dois métodos divergem totalmente.

Para o caso do campus da UNESP (Bauru) vários ensaios foram testados e muito pouco pôde ser feito em relação à determinação da curva de dispersão dos dados da área. Tentou-se obter uma curva de dispersão média que fosse representativa do meio, mas pela comparação com os dados obtidos por "crosshole", notou-se que este procedimento foi totalmente infrutífero, uma vez que os valores obtidos não são em nenhuma profundidade semelhantes os valores determinados pelos crosshole. Portanto, conclui-se que o método SASW, utilizando fontes portáteis, conforme proposto no início deste trabalho, não é aplicável a profundidades até $20 \mathrm{~m}$. No entanto, dependendo da área, pode ser utilizado para profundidades menores. A definição da profundidade de investigação dependerá da qualidade dos sinais de baixa freqüência, bem como da razão sinal-ruído. Deve-se ressaltar ainda que, considerando a sensibilidade do método a baixas relações sinal ruído, sua aplicação em ambientes urbanos fica seriamente comprometida.

\section{Agradecimentos}

À Fundação de Amparo à Pesquisa do Estado de São Paulo - FAPESP pela bolsa de estudos e respectivo apoio financeiro, através do Processo 96/11.969-5.

Aos docentes, colegas e funcionários do IG-USP, IAGUSP, UNESP e UNICAMP

\section{Referências}

CARVALHO, D.; ALBUQUERQUE, P. J. R.; GIACHETI, H. L. (2000) Campo experimental para estudos de mecânica dos solos e fundações em Campinas-SP, Seminário de Engenharia de Fundações Especiais e Geotecnia, SEFE IV, V. 3, p. 90-100.

FOTI, S. (2000) Multistation Methods for Geotechnical Characterization using Surface Waves. PhD Thesis . Politecnico di Torino, pp.229;

NAZARIAN, S.; STOKOE II, K. H.; HUDSON, W. R.

(1983) Use of Spectral Analysis of Surface Waves

Method for Determination of Moduli and Thicknesses of Pavement Systems, Transportation Research Record 930, pp.38-45.

NAZARIAN, S.; STOKOE II, K. H. (1986) Use of Surface Waves in Pavement Evaluation. Transportation Research Record 1070, pp.132-144

NAZARIAN, S.; DESAI, M. R. (1993) Automated Surface Wave Method: Field Testing, Journal Geotechnical Engineering, ASCE, 119(7). Pp. 1094-1111.

PARK, C.B.; MILLER, R.B.; XIA, J. (1999a) Multi-channel analysis of surface waves. Geophysics, v.64, 800-807. PARK, C.B.; MILLER, R.B.; XIA, J. (1999b) Multimodal analysis of high frequency surface waves. Proceedings of the Symposium on the Application of Geophysics to Engineering and Environmental Problems (SAGEEP 99), Oakland, CA, March 14-18, pp. 115-122.

SANCHES-SALINERO, I.; ROESSET, J. M.; SHAO, K. Y.; STOKOE II, K. H. ; RIX, G. J. (1987). Analytical Evaluation of Variables Affecting Surface Wave Testing of Pavements. Transportation Research Record 1136, pp. 86-95.

TOKIMATSU, K.; TAMURA, S.;KOJIMA, H., (1992) Effects of Multiple Modes on Rayleigh Wave Dispersion Characteristics. Journal of Geotechnical Engineering, Volume 118, Number 10: 1529-1543

XIA, J,; MILLER, R.D.; PARK, C. B.; HUNTER, J. A.; HARRIS, J.B. (2000) Comparing shear wave velocity profiles from MASW with borehole measurements in unconsolidated sediments, Fraser River Delta, B.C., Canada. Journal of Environmental \& Engineering Geophysics. V. 5, pp. 1-14.

ZYWICKI, D.J. (1999) Advanced signal processing methods applied to engineering analysis of seismic surface waves. PhD. Thesis . Georgia Institute of Technology, pp.227. 